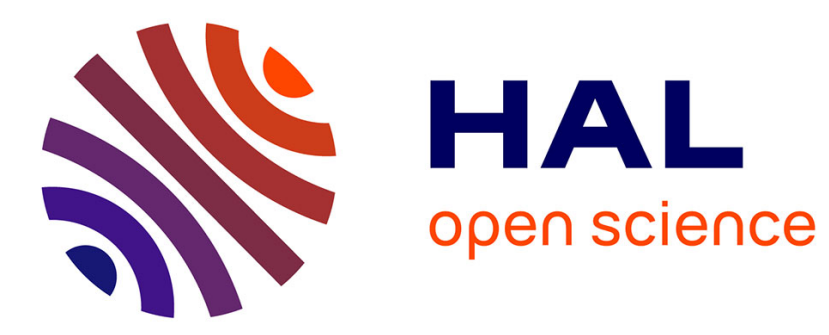

\title{
TESTS OF DYNAMICS THROUGH POLARIZATION EXPERIMENTS : THE CASE OF THE DIBARYON
}

\author{
G. Goldstein, M. Moravcsik
}

\section{To cite this version:}

G. Goldstein, M. Moravcsik. TESTS OF DYNAMICS THROUGH POLARIZATION EXPERIMENTS : THE CASE OF THE DIBARYON. Journal de Physique Colloques, 1985, 46 (C2), pp.C2353-C2-362. 10.1051/jphyscol:1985241 . jpa-00224555

\section{HAL Id: jpa-00224555 https://hal.science/jpa-00224555}

Submitted on 1 Jan 1985

HAL is a multi-disciplinary open access archive for the deposit and dissemination of scientific research documents, whether they are published or not. The documents may come from teaching and research institutions in France or abroad, or from public or private research centers.
L'archive ouverte pluridisciplinaire HAL, est destinée au dépôt et à la diffusion de documents scientifiques de niveau recherche, publiés ou non, émanant des établissements d'enseignement et de recherche français ou étrangers, des laboratoires publics ou privés. 
JOURNAL DE PHYSIQUE

Colloque $\mathrm{C} 2$, supplément au $\mathrm{n}^{\circ} 2$, Tome 46 , février 1985

page $\mathrm{C} 2-353$

TESTS OF DYNAMICS THROUGH POLARIZATION EXPERIMENTS : THE CASE OF THE DIBARYON

G.R. Goldstein and M.J. Moravcsik*

Department of Physics, Tufts University, Medford, Mass. 02155, U.S.A.

Institute of Theoretical Science, University of Oregon, Eugene, Oregon 97403, U.S.A.

Résumé - Nous présentons une revue du formalisme optimal pour relier les amplitudes et les observables dans la diffusion à deux corps. Ce formalisme est appliqué au problème de la détermination complète d'ensembles non ambigüs d'amplitudes; il conduit au fait que le vecteur polarisation est superflu pour déterminer des amplitudes du deuteron et au fait que des états dibaryon singlet et triplet sont plausibles dans la diffusion élastique p-p.

Abstract - The Optimal Formalism for relating amplitudes and observables in two-body scattering is reviewed. It is applied to : the problem of determining complete unambiguous sets of amplitudes; the redundancy of vector polarization for determining deuteron amplitudes ; the plausibility of singlet and triplet dibaryon states in p-p elastic scattering.

\section{Introduction}

The underlying dynamics of particle interactions is surely reflected in the spin dependence of scattering processes. The long history of particle. physics confirms this as does the shorter history of this symposium. From the discovery of parity violation to the confirmation of the Electroweak Standard Model, polarization experiments have been crucial to our theoretical progress. The enormous effort and expense involved in initiating and completing polarization studies has been rewarded with increasing understanding of dynamics. However, it is not always clear that our existing theoretical notions about dynamics provide adequate explanations of al1 polarization phenomena.

Experiments often leave us with more puzzles than we knew we had. For this reason it is important to be able to analyse polarization phenomena in a general way unprejudiced by particular dynamical schemes. For interpreting 
existing data, for finding new tests of dynamical models and for designing future experiments it is crucial to have a framework in which the relation between observable quantities and underlying spin dependence can be spectfied, in a model independent way.

Over the last several years we have developed and applied a formalism that relates the spin dependent amplitudes for a two-body reaction to observable quantities in the simplest possible way.(1-4) This "0ptimal Formalism" allows us to study general abstract questions about amplitude structure(1) under various symmetry constraints $(2)$ and the corresponding observable structure. The freedom in choosing different frames in which to specify amplitudes and observables leads to an exploration of the consequences of those cholces for testing different dynamical hypotheses. We have used the Optimal Formalism to explore many problems, both abstract and practical. (4)

Hereln we will discuss several formal applications of this framework and a particular application to the dynamics of $p-p$ scattering. We have recently answered the question of what constitutes a minlmal set of experiments from which to determine the amplitudes completely unambiguously.(3) We w111 review this work. A second interesting application involves the tensor polarization of the deuteron.(5) Finally we discuss the amplitude structure for $p-p$ scattering in the region of the presumed dibaryon resonances. (6) Optima 1 Forma1tsm

The Optimal Formalism has been discussed in considerable deta11 elsewhere $(1-4)$ and will be reviewed only briefly here. Consider a two-body reaction $A+B+C+D$ for which the spins are $S^{A}, S^{B}, S^{C}$, and $S^{D}$. Now the spin dependent amplitudes are defined in terms of the spin quantization along some particuar directions in space for each of the four particles. Let $a, b, c, d$ be the expectation values of the spins with respect to the axes labelled

$\wedge A \wedge B \wedge C \wedge D$ i.e.

$z, z, z, z$,

$$
a=\left\langle\vec{S} A \cdot \hat{Z^{A}}\right\rangle, \quad b=\left\langle\vec{S} \mathrm{~B} \cdot \hat{Z^{B}}\right\rangle, \cdots
$$


The amplitudes then can be written as $F_{C, d ; a, b}(E, \theta)$ in an obvious notation. These axes are specified independently - For conventence they are specifled in the Center-of-Mass frame of the reaction. There is a continuum of possibilities for these axes, although symmetries restrict those cholces somewhat (2) as follows.

Parity conservation requires that the axes be either normal to the scattering plane or $\underline{\text { In }}$ the plane in order that the amplitudes transform into one another under the parity operation. Time reversal invariance requires that the $\hat{z^{A}}$ and $\hat{z^{C}}$ be parallel as well as the $\hat{z^{B}}$ and $\hat{z}^{D}$ axes. Identical particle constraints require $\hat{z^{A}}$ parallel to $\hat{z^{B}}$ and $\hat{z^{C}}$ parallel to $\hat{z}^{D}$. So for parity conservation alone, which will be assumed in a11 applications considered here, each particle's axis can be specified by the angle that axis makes with the particle's momentum direction when the axis lies in the scattering plane. If only parity is relevant then, four angles parameterize the four axes in the plane. This choice of axes is called a "Planar Frame" and the corresponding amplitudes are "Planar Amplitudes". Alternatively, of course, any of the axes may be transverse to the plane. When all axes are transverse the amplitudes are "Transversity Amplitudes".

With these restrictions the nucleon axes in pion-photoproduction, for example, can be chosen either in the scattering plane with two independent angles or both normal to that plane.(7) On the other hand the axes for the protons in p-p elastic scattering, if they are chosen planar, must all be at the same angle with respect to their momenta.(2) There is only on choice of orientation to be made and hence one angle to specify.

Which of the infinitude of axes are chosen depends on the application anticipated. For extracting amplitudes from observables the transversity axes are usually most convenient. Single particle polarization asymmetry is then a combination of squares of magnitudes of transversity amplitudes. For making model predictions the helicity frame has been preferred because of the simplicity of the helicity dependence of most hypothetical interactions at high energies. 
The helicity frame is just a special case of the planar frame for which a 11 angles are zero.

Given a set of transversity or helicity amplitudes extracted from data for some reaction, it is an easy matter to obtain planar amplitudes for any particular cholce of orientation angles. Choosing those angles propitiously may reveal some hitherto unexpected structure reflecting new dynamics. In the p-p elastic system this exploration has been done for those energles and scattering angles at which a complete set of polarization data exist. (8) For the $6 \mathrm{GeV} / \mathrm{c}$ and the 300 to $800 \mathrm{MeV}$ data a remarkable simplictty results when the planar frame is chosen with each axis at $90^{\circ}$ to the corresponding momentum. In this Planar-Transversity or "Sideways" frame the amplitudes are almost entirely pure real or pure lmaginary with respect to one another. Why this should be is not clear but certainly suggests some underlying simplicity not incorporated In any conventional dynamical model. (9)

Having defined frames and amplitudes the observables can now be specified.(1) The simplest structure is obtained by choosing Inftial state particle density matrices to have single entries on the diagonal or two off-diagonal elements to insure hermiticity. Similarly the final state polarizations are traces of final state density matrices with spin operators that are chosen with one diagonal entry or two off-diagonal entries. For spin $1 / 2$ these matrices are

$$
\left(\begin{array}{ll}
1 & 0 \\
0 & 0
\end{array}\right),\left(\begin{array}{ll}
0 & 0 \\
0 & 1
\end{array}\right),\left(\begin{array}{ll}
0 & 1 \\
1 & 0
\end{array}\right),\left(\begin{array}{lr}
0 & -1 \\
1 & 0
\end{array}\right)
$$

These correspond to complete polarization along the z-axis (purely + or $1 / 2$ ), or asymmetries along $x$ - or y-axes. For spin-1 the sttuation is more complicated, $(1,2,5)$ involving both vector and tensor polarizations as indicated here:

$$
\begin{aligned}
& \left(\begin{array}{ccc}
1 & 0 & 0 \\
0 & 0 & 0 \\
0 & 0 & 0
\end{array}\right) \sim \mathrm{s}_{z}+\mathrm{s}_{z} \mathrm{~s}_{z},\left(\begin{array}{ccc}
0 & 1 & 0 \\
1 & 0 & 0 \\
0 & 0 & 0
\end{array}\right) \sim \mathrm{s}_{x}+\left\{\mathrm{s}_{\mathrm{x}}, \mathrm{s}_{z}\right\} \\
& \left(\begin{array}{ccc}
0 & -1 & 0 \\
1 & 0 & 0 \\
0 & 0 & 0
\end{array}\right) \sim \mathrm{s}_{y}+\left\{\mathrm{s}_{\mathrm{y}}, \mathrm{s}_{z}\right\},\left(\begin{array}{ccc}
0 & 0 & 1 \\
0 & 0 & 0 \\
1 & 0 & 0
\end{array}\right) \sim \mathrm{s}_{x} \mathrm{~S}_{x}-\mathrm{S}_{y} \mathrm{~s}_{y},
\end{aligned}
$$




$$
\left(\begin{array}{ccc}
0 & 0 & -1 \\
0 & 0 & 0 \\
1 & 0 & 0
\end{array}\right)-\left\{s_{x}, s_{y}\right\}, \cdots
$$

Note that matrices with imaginary components correspond to odd numbers of $s_{y}$ 's. Each matrix is a linear combination of scalar and/or vector with tensor polartzations. As observables they are conceptually as simple as vector and tensor polarizations. They correspond to various comblnations of pure spin components $+1,0,-1$ along the z-axis, the x-axis, the y-axis, $45^{\circ}$ axis in the $\mathrm{x}-\mathrm{z}$ plane, etc. In practice, of course, not all particles are in prepared or measured polarization states. Actual observables are sums of these optima 1 choices. Nevertheless considerable insight into both formal and practical applications is obtained from study of these optimal observables as the examples below indicate.

Forma1 applications

The Optimal Formalism allows us to answer a simple and very fundamental quantum maechanical question. For a particular two body reaction, what types of measurements are necessary for completely determining the underlying amplitudes unambiguously? Without any symmetry restrictions we showed previously that measurements of all the spin profections of each particle's spin along two Independent directions were necessary to completely determine the amplitudes. (4) of course those measurements must include at least double correlations with other particle's spins.(10) This specification, however, allows discrete ambiguities to remain in the amplitudes.

To remove discrete ambiguities as well it is necessary to measure at least one observable with spin polarization along three independent directions. The proof is straightforward using the optimal formalism. (3) Measuring or preparing one particle's polarization along a single direction involves diagonal elements of the density matrix for that particle (in the basis for which that direction is the quantization direction). Hence the corresponding observables involve sums of squares of magnitudes. Such 
observables can yield the magnitudes but not relative phases of those a mplitudes.

Polarization along a second orthogonal direction gives observables involving the sum of real (or imaginary, depending on convention) parts of bilinear products of amplitudes. From these observables the cosines of relative phases between amplitudes is determined. But cosines leave sign ambiguities in the resulting phase angles.

It is finally necessary then to measure or prepare at least some polarizations along a third orthogonal direction. This involves observables that are sums of imaginary parts of bilinear products of amplitudes. Those observables therefore determine the sines of some of the same phase angles and, thereby, remove the remaining quadrant ambiguities.

This sketch of the proof shows the utility of the formalism and indicates why it is a valuable tool for exploring such general questions.

Another formal application to deuteron polarization leads to a dramatic and unexpected result. We have recently shown that in reactions involving deuterons (or any spin-1 particles) al1 amplitude information can be obtained by measuring or preparing only tensor polarizations of the deuterons. (5) Whether or not this has some practical significance remains to be seen, but it does alter one's intuition about spin-1. We will present a plausibility argument here; the proof being too lengthy.

Consider the simple reaction involving spins $0+0 \rightarrow 0+1$ and assume on $1 y$ Lorentz invariance. There are 3 complex amplitudes, A,B,C corresponding to 3 values of the spin projections along some axis to be chosen as the z-axis. With 3 amplitudes, 5 observables are needed from which to specify the magnitudes and 2 relative phases of the amplitudes.

Choose the observables to be the differential cross section and 4 of the 5 independent tensor polarizations defined here in terms of the bilinear products of the amplitudes: 


$$
\begin{aligned}
& \sigma^{\sim}|A|^{2}+|B|^{2}+|C|^{2} \\
& p_{z z} \sim|A|^{2}+|C|^{2}-2|B|^{2} \\
& p_{x z} \sim \operatorname{Re}\left(B C^{*}-A B^{*}\right) \\
& p_{y z} \sim \operatorname{Im}\left(B C^{*}-A B^{*}\right) \\
& p_{x x^{-}}-p_{y y}{ }^{\sim} \operatorname{Re}\left(A C^{*}\right) \\
& p_{x y} \sim \operatorname{Im}\left(A C^{*}\right)
\end{aligned}
$$

From $\sigma$ and $P_{z z}$ the quantities $|A|^{2}+|C|^{2}$ and $|B|$ are determined while $P_{x x}-P_{y y}$ and $P_{x y}$ then fix A and $C$ (choosing A real say). Then only $P_{x z}$ or $P_{y z}$ suffices to specify $B$. When more spinning particles are involved in the process there are complications (which have been elucidated in ref. 5) but the generalization goes through by including those same tensor measurements in correlation with other spin polarizations.

This theorem could have significance in the pursuit of more experimental information on the inelastic channels in $p-p$ and $\pi-d$ scattering wherein dibaryons may be prominant. This leads to our last application here--dibaryons in $p-p$ elastic scattering amplitudes. The Case of the Dibaryon

We are going to consider how the elastic amplitudes reflect the existence of resonances with various spin-angular momentum quantum numbers. For this application helicity amplitudes prove most convenient; they reflect the symmetries in $\mathrm{p}-\mathrm{p}$ elastic scattering most directly when decomposed into definite angular momentum states.

The total angular momentum decomposition of helicity amplitudes(11)

$$
\text { Dhelicity }(c, d ; a, b)=\underset{J}{\sum}\langle J(c, d) \mid J(a, b)\rangle d_{a-b} J-c-d(\theta)
$$

can be separated into spin singlet and triplet combinations for the p-p system. Recall that singlets must have even $\mathrm{L}$ and triplets odd $\mathrm{L}$ (and $\mathrm{J}=\mathrm{L}+\mathrm{l}$, $\mathrm{L}, \mathrm{L}-1)$ due to identical particle symmetry. Then the helicity amplitude decompositions become

$$
\left.\left.\left.\begin{array}{l}
\mathrm{a}=\mathrm{D}(+,+;+,+) \\
\mathrm{d}=\mathrm{D}(+,+;-,-)
\end{array}\right\}=\left.\sum_{\mathrm{J}}^{\sum \mathrm{d}_{0}^{\mathrm{J}}(\theta)\left\{\left\langle^{3}(\mathrm{~J} \pm 1), 0\right.\right.}\right|_{\mathrm{J}} ^{3}(\mathrm{~J} \pm 1)_{\mathrm{J}}, 0\right\rangle \pm\left\langle\mathrm{l}_{\mathrm{J}},\left.0\right|^{1} \mathrm{~J}_{\mathrm{J}}, 0\right\rangle\right\},
$$




$$
\begin{aligned}
& \left.\begin{array}{l}
\mathrm{c}=\mathrm{D}(+,-;+,-) \\
\mathrm{e}=\mathrm{D}(+,-;-,+)
\end{array}\right\}=\sum_{\mathrm{J}} \mathrm{d}_{1_{ \pm}}(\theta)\left\{\left\langle^{3}(\mathrm{~J} \pm 1)_{\mathrm{J}}, \pm\left. 1\right|^{3}(\mathrm{~J} \pm 1)_{\mathrm{J}}, \pm 1\right\rangle \pm\left\langle{ }^{3} \mathrm{JJ}, \pm\left. 1\right|^{3} \mathrm{~J}_{\mathrm{J}}, \pm 1\right\rangle\right\}, \\
& \mathrm{b}=\mathrm{D}(+,+;+,-)=\sum_{\mathrm{J}} \mathrm{d}_{01}(\theta)\left\langle^{3}(\mathrm{~J} \pm 1)_{\mathrm{J}}, \pm\left. 1\right|^{3}(\mathrm{~J} \pm 1)_{\mathrm{J}}, 0\right\rangle, \\
& \text { with notation } \mathrm{S}(\mathrm{L})_{\mathrm{J}} \text { for the states. }(12)
\end{aligned}
$$

Note that for a ${ }^{1} J_{J}$ or ${ }^{3}(J \pm 1) J$ resonance the amplitudes $a=-o r+d$. However, for ${ }^{3} \mathrm{~J}_{\mathrm{J}}$ or ${ }^{3}(\mathrm{~J} \pm 1) \mathrm{J}$ c $\neq-$ orte because of the different angular functions involved. There are relations in the latter case nevertheless; they are dependent on angle as we shall see below. The amplitudes thus provide a means to test for the existence of resonances by observing the amplitude relations as energy varies. (6)

Complete sets of amplitudes do not exist for all energies and angles in the presumed dibaryon region since complete sets of experiments have been performed only at select kinematic values. Phase shift analyses have been performed in the range of interest--here 700 to $900 \mathrm{MeV}$. To the extent that phase shifts are believable they interpolate experimental data of various kinds by using unitarity and the assumption of a finite range interaction (i.e. Lmax). Using one of the "standard" phase shift analyses, the SAID set(13), we can determine the amplitudes a,...,e.

The resulting amplitudes can be studied for their resonance structure as outlined. The magnitudes of a and d are quite different and show no indication of approaching one another as the energy goes through the presumed mass of the ${ }^{l_{D}}{ }_{2}$ state. The phase difference between a and $d$ exhibits no special changes as the same region is studied. There is no sign of resonance activity in these amplitudes indicating either a dominant background contribution or no real effect.

The ${ }^{3} F_{3}$ state escapes notice in the above test. Examining the angular dependence of $|c|$ and $|e|$, however, shows a striking behavior as the figure below exhibits. The magnitudes are equal at $90^{\circ}$, where they are so constrained. But they are also equal at somewhat smaller angles; there is a crossover. And that crossover point moves to smaller angles as the energy 
increases. Remember that $c^{\sim} \mathrm{d}_{11}^{J}(\theta)$ and $\mathrm{e}^{\sim} \mathrm{d}_{1_{-1}}^{\mathrm{J}}(\theta)$. If $J=3$ dominates these amplitudes then at those angles for which $d_{11}^{J}(\theta)=-d_{1}^{J}(\theta)$ the values of $c \cong-$ This equality occurs for $63^{\circ}$ which is close to the crossover angle at $800-850$ $\mathrm{MeV}$ as the figure shows. Furthermore note that $\mathrm{d}_{11}^{\mathrm{J}}(\theta)=0$ at $85^{\circ}$ so that $\mathrm{c}$ should be zero there. It is far from zero showing that there is sizeable background contributing. In spite of that background there is the striking appearance and energy dependent behavior of that crossover to verify a resonant contribution. In addition the relative phase between $c$ and e varies rapidly through $180^{\circ}$ near the $63^{\circ}$ scattering angle. The case for the ${ }^{3} \mathrm{~F}_{3}$ is

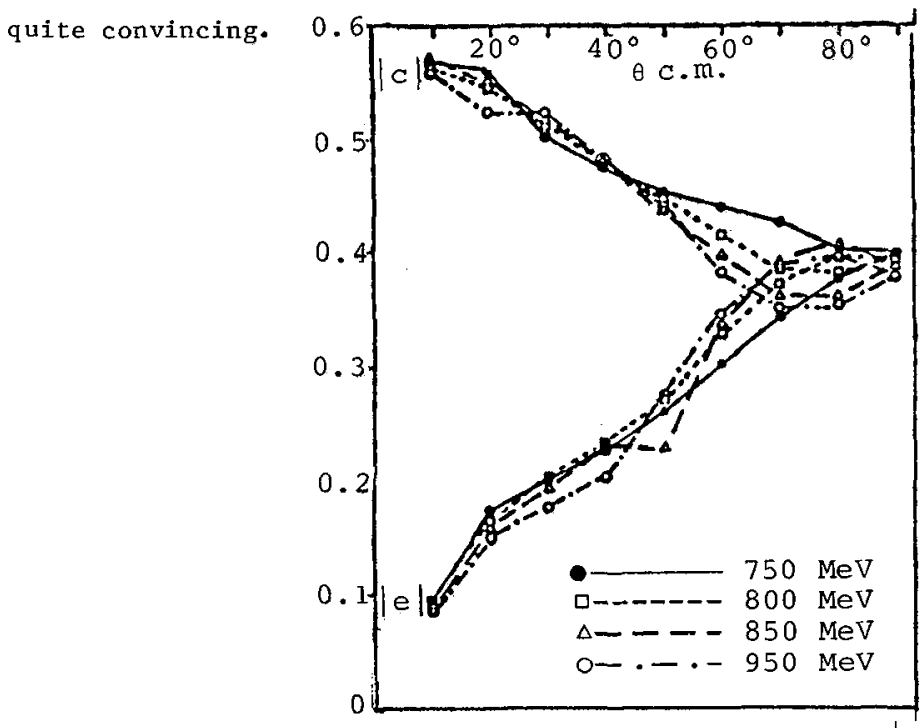

To summarize this amplitude test we emphasize that we have used the phase shifts only to represent interpolation and extrapolation of cross section and polarization data to various angles and energies. The amplitudes that emerge are an equivalent interpolation-extrapolation of data. Those amplitudes show smooth "uninteresting" behavior for the singlet spin amplitudes. Therefore observables will not show any "interesting" behavior with energy (even though the singlet phase shift, ${ }^{1} D_{2}$, loops on the Argand diagram ${ }^{13}$ ). The question of whether or not this looping partial wave is truely unique arises. Where is the energy variation in an observable quantity or amplitude that shows such a singlet resonance? Of course there needn't be such a variation in any particular set of observables to still give a resonating partial wave so long 
as subtle cancelations occur. And large inelasticities muddy the situation. In contrast however, the $3 \mathrm{~F}_{3}$ state is far more plausible because of the crossover phenomenum observed above.

To conclude, we have demonstrated the utility of the optimal formalism by consldering three very distinct applications. In each case somewhat surprising results were presented to illustrate the Improved intuition this amplitude formalism produces. The formalisim has many more applications, some of which were summarized in the High Energy Workshop of this Sumposium. It is anticipated that the insights gained through this approach will continue to prove advantageous in both the design of experimental programs for polarization measurements and the interpretation of the resulting data.

We acknowledge the support provided by grants from the U.S. Department of Energy. We are grateful to the organizers of the Symposium for their exceptional help; particularly Jacques Soffer.

\section{$\underline{\text { References }}$}

1. G.R. Goldstein and M.J. Moravesik, Ann. Phys. (N.Y.) 98, 128 (1976).

2. G.R. Goldstein and M.J. Moravcsik, Ann. Phys. (N.Y.) 142, 219 (1982).

3. K. Nam, M.J. Moravcsik, and G.R. Goldstein, Phys. Rev. Letters, 52 2305 (1984).

4. G.R. Goldstein and M.J. Moravcsik, Ann. Phys. (N.Y.) 126, 176 (1980).

5. G.R. Goldstein and M.J. Moravcsik, "Vector Polarlaation in Reactions with Spin-1 Particles" Univ, of Oregon preprint OITS 252 (1984).

6. G.R. Goldstein and M.J. Moravcsik, and F. Arash, "Amplitude Tests of Direct Channel Resonances: the Dibaryon" Univ. of Oregon preprint OITS 235 (1984)

7. G.R. Goidstein and M.J. Moravcsik, Nuov. C1m. 73A, 196 (1983).

8. M.J. Moravcsik, F. Arash, and G.R. Goldstein, "A Striking Pattern in a Strong Interaction Reaction", Univ, of Oregon preprint OITS 280 (1984).

9. G.R. Goldstein and M.J. Moravcsik, "Exploration of Dynamics Through Polarization Experiments" contribution to High Energy Workshop, this Symposium.

10. M. Simonius, Phys. Rev. Letters 19, 279 (1967).

11. M. Jacob and G.C. Wick, Ann. Phys. (N.Y.) 7, 404 (1959).

12. G.R. Goldstein and M.J. Moravcsik, "The Optima1 Polarization Formalism and Partial Waves" Unfv. of Oregon preprint OITS 225 to be published in Nuov. C1m. (1984).

13. R.A. Arndt et a1, Phys, Rev. D28, 97 (1983). 